\title{
COVID-19 Pneumonia: A Potential Role of Homeopathy
}

\author{
Alfonso Tramontana ${ }^{1}$ \\ ${ }^{1}$ Department of Physical and Rehabilitation Medicine, University Rey \\ Juan Carlos, Calle Tulipán, Móstoles, Madrid, Spain \\ Homeopathy $2021 ; 110: 70-71$.
}

I am pleased to submit for your attention this letter about the potential employment of homeopathy in the treatment of coronavirus disease 2019 (COVID-19). This observation stems from the evaluation of meaningful evidence: one of the main alterations in patients with COVID-19 is interstitial pneumonia with an abnormal hyperergic response of the immune system. This specific kind of pneumonia is the regular viral infection of the lungs and it is not a distinguishing type derived from a new kind of virus. ${ }^{1}$ In fact, considering the pathology classification of pneumonia processes, it is clear that there are significant factors which set apart a bacterial pneumonia and a viral pneumonia. Bacterial pneumonia produces acute alveolar damage and alveolar infiltration; on the other hand, viral pneumonia is always characterized by interstitial infiltration and it compromises the compliance function of the lung. In patients with COVID-19, the typical data show a hyperergic immune response and not an interstitial inhibition of the lung. In many cases, the conventional therapies administered until now have not improved the interstitial edema. It is still not clear how to produce an effective and safe immunosuppressive effect to treat the hyperergic pathological immune response due to COVID-19. The strategy for treating patients who exhibit such symptoms is not clear-cut.

In examining the state-of-the-art scientific literature, there is a dispute over an absolute treatment that would work for almost everyone. For example, some studies have highlighted relevant side effects in patients who have been affected after receiving immunosuppressors and interleukin-based therapies. ${ }^{2-6}$ One of these side effects is cytokine release syndrome, which refers to an uncontrolled and overwhelming release of pro-inflammatory mediators by an overly activated immune system. Other authors underline that in most cases anti-viral therapy alone may be inadequate. Therapies based on corticosteroids and serotherapy are not clearly stated. More than half of SARS

received

August 27, 2020

accepted after revision

September 1, 2020

published online

December 21, 2020
Address for correspondence Alfonso Tramontana, MD, PhD, Department of Physical and Rehabilitation Medicine, University Rey Juan Carlos, Calle Tulipán, s/n, 28933 Móstoles, Madrid, Spain (e-mail: altramontana75@gmail.com).

patients treated with corticosteroids suffer from joint pain and bone marrow abnormalities. Other therapies aiming to dampen excessive serum inflammatory mediators, such as plasmapheresis or continuous renal replacement therapy, either require specific equipment or lack documented efficacy. An important limitation to the administration of the therapies tested so far is that they do not always interact well with the drugs that patients with pre-existing medical conditions were taking.

I am specialized in Thoracic Surgery and Physical \& Rehabilitation Medicine. I have treated many interstitial pneumonia cases and I also studied their anatomical pathology in cadaveric dissections. ${ }^{7}$ During these years of clinical practice, I integrated three homeopathic medicines, Arsenicum album, Stannum, and Ribes nigrum. When treating patients with such a disease, I found that they were quite beneficial. The patients with interstitial restrictive pneumonia who did not respond well to previous conventional therapy improved greatly, both in subjective symptoms and functional respiratory outcomes. The rational use of these remedies could be effective, ${ }^{8}$ considering that Ribes produces an effect similar to cortisone and can reduce both the inflammatory response and the hyperergic immune response. According to materia medica reports on Stannum, "Debility is very marked when Stannum is the remedy, especially the debility of chronic bronchial and pulmonary conditions, characterized by profuse muco-purulent discharges". And on Arsenicum album: "Air passages constricted. Asthma worse at night. Burning in chest. Suffocative catarrh. Cough worse after midnight; worse if lying on the back. Expectoration. Wheezing respiration".

In conclusion, in the hopes that my letter might evoke an experimental clinical trial, my suggestion is that the association of these homeopathic medicines with conventional therapies may potentially improve the pneumonia in COVID19 non-responder patients, especially in the early stages of the disease.

\footnotetext{
(c) 2020. The Faculty of Homeopathy.

All rights reserved.

Georg Thieme Verlag KG,

Rüdigerstraße 14,

70469 Stuttgart, Germany
}

DOI https://doi.org/ 10.1055/s-0040-1721063. ISSN $1475-4916$. 


\section{Conflict of Interest}

The author declares no conflict of interest with any financial organization regarding the topic discussed in the manuscript.

\section{References}

1 Ruuskanen O, Lahti E, Jennings LC, Murdoch DR. Viral pneumonia. Lancet 2011;377:1264-1275

2 Liu B, Li M, Zhou Z, Guan X, Xiang Y. Can we use interleukin-6 (IL6) blockade for coronavirus disease 2019 (COVID-19)-induced cytokine release syndrome (CRS)? J Autoimmun 2020;111; 102452

3 Neelapu SS, Tummala S, Kebriaei P, et al. Chimeric antigen receptor T-cell therapy-assessment and management of toxicities. Nat Rev Clin Oncol 2018;15:47-62
4 Peiris JS, Chu CM, Cheng VC, et al; HKU/UCH SARS Study Group. Clinical progression and viral load in a community outbreak of coronavirus-associated SARS pneumonia: a prospective study. Lancet 2003;361:1767-1772

5 Wang D, Hu B, Hu C, et al. Clinical characteristics of 138 hospitalized patients with 2019 novel coronavirus-infected pneumonia in Wuhan, China. JAMA 2020;323:1061-1069

6 Bersanelli M. Controversies about COVID-19 and anticancer treatment with immune checkpoint inhibitors. Immunotherapy 2020;12:269-273

7 Tramontana A, Pavia R, Reina De la Torre F, et al. Bronchial arteries: anatomical and clinical studies and strategies in surgical approach [article in Italian]. Minerva Chir 2004;59:307-311

8 Tramontana A. Is homoeopathy effective or are its effects similar to placebo? A critical analysis of systematic reviews on this topic. Gazzetta medica Italiana 2017;176:202-207 